\title{
Difference methods for the Darboux problem for functional partial differential equations
}

\author{
by Tomasz CzŁapiński (Gdańsk)
}

Abstract. We consider the following Darboux problem:

$$
\begin{aligned}
& D_{x y} z(x, y)=f\left(x, y, z_{(x, y)},\left(D_{x} z\right)_{(x, y)},\left(D_{y} z\right)_{(x, y)}\right), \\
& z(x, y)=\phi(x, y) \quad \text { on }\left[-a_{0}, a\right] \times\left[-b_{0}, b\right] \backslash(0, a] \times(0, b],
\end{aligned}
$$

where $a_{0}, b_{0} \in \mathbb{R}_{+}, a, b>0$. The operator $[0, a] \times[0, b] \ni(x, y) \mapsto \omega_{(x, y)} \in C\left(\left[-a_{0}, 0\right] \times\right.$ $\left.\left[-b_{0}, 0\right], \mathbb{R}\right)$ defined by $\omega_{(x, y)}(t, s)=\omega(t+x, s+y)$ represents the functional dependence on the unknown function and its derivatives. We construct a wide class of difference methods for problem (1),(2). We prove the existence of solutions of implicit functional systems by means of a comparative method. We get two convergence theorems for implicit and explicit schemes, in the latter case with a nonlinear estimate with respect to the third variable. We give numerical examples to illustrate these results.

1. Introduction. Given $a, b>0$ and $a_{0}, b_{0} \in \mathbb{R}_{+}=[0, \infty)$, we define

$$
E=(0, a] \times(0, b], \quad E^{0}=\left[-a_{0}, a\right] \times\left[-b_{0}, b\right] \backslash(0, a] \times(0, b],
$$

and $B=\left[-a_{0}, 0\right] \times\left[-b_{0}, 0\right]$. For any function $\omega: E^{0} \cup E \rightarrow \mathbb{R}$ and $(x, y) \in E$ we define $\omega_{(x, y)}: B \rightarrow \mathbb{R}$ by

$$
\omega_{(x, y)}(t, s)=\omega(x+t, y+s), \quad(t, s) \in B .
$$

Consider the following Darboux problem for a second order functional partial differential equation:

$$
\begin{aligned}
D_{x y} z(x, y) & =f\left(x, y, z_{(x, y)},\left(D_{x} z\right)_{(x, y)},\left(D_{y} z\right)_{(x, y)}\right), \quad(x, y) \in E, \\
z(x, y) & =\phi(x, y), \quad(x, y) \in E^{0},
\end{aligned}
$$

where $f: E \times C(B, \mathbb{R})^{3} \rightarrow \mathbb{R}$ and $\phi \in C^{1}\left(E^{0}, \mathbb{R}\right)$ are given functions. We call $z \in C^{1}\left(E^{0} \cup E, \mathbb{R}\right)$ a solution of (1), (2) if $z$ has a continuous mixed derivative and satisfies (1) on $E$, and $z$ fulfills the Darboux condition (2) on $E^{0}$. In

1991 Mathematics Subject Classification: 35G30, 35L70, 35R10.

Key words and phrases: functional differential equation, Darboux problem, classical solutions, difference method, comparative method. 
other words we will consider difference methods for classical solutions of problem (1), (2). A model of functional dependence in (1), (2) based on the operator $(x, y) \mapsto \omega_{(x, y)}$ contains as particular cases differential equations with deviated argument and integral-differential problems.

ExAmple 1. Consider the differential equation with deviated argument

$$
D_{x y} z(x, y)=\widetilde{f}\left(x, y, z\left(\gamma_{0}(x, y)\right), D_{x} z\left(\gamma_{1}(x, y)\right), D_{y} z\left(\gamma_{2}(x, y)\right)\right),
$$

where $\tilde{f}: E \times \mathbb{R}^{3} \rightarrow \mathbb{R}$ and $\gamma_{0}, \gamma_{1}, \gamma_{2}: E \rightarrow E^{0} \cup E$ are given functions such that $\gamma_{i}(x, y)-(x, y) \in B, i=0,1,2$, for $(x, y) \in E$. If we define $f$ by

$f\left(x, y, w, w_{1}, w_{2}\right)$

$$
=\widetilde{f}\left(x, y, w\left(\gamma_{0}(x, y)-(x, y)\right), w_{1}\left(\gamma_{1}(x, y)-(x, y)\right), w_{2}\left(\gamma_{2}(x, y)-(x, y)\right)\right)
$$

for $\left(x, y, w, w_{1}, w_{2}\right) \in E \times C(B, \mathbb{R})^{3}$ then (3) becomes a special case of (1).

EXAMPLE 2. The differential-integral equation

$$
\begin{aligned}
& D_{x y} z(x, y)=\tilde{f}\left(x, y, \int_{B} G_{0}(t, s, z(x+t, y+s)) d t d s,\right. \\
& \left.\int_{B} G_{1}\left(t, s, D_{x} z(x+t, y+s)\right) d t d s, \int_{B} G_{2}\left(t, s, D_{y} z(x+t, y+s)\right) d t d s\right),
\end{aligned}
$$

where $\tilde{f}: E \times \mathbb{R}^{3} \rightarrow \mathbb{R}$ and $G_{0}, G_{1}, G_{2}: B \times \mathbb{R} \rightarrow \mathbb{R}$ are given functions, is also a special case of (1) if we take

$$
\begin{aligned}
f\left(x, y, w, w_{1}, w_{2}\right)=\tilde{f}(x, y, & \int_{B} G_{0}(t, s, w(t, s)) d t d s, \\
& \left.\int_{B} G_{1}\left(t, s, w_{1}(t, s)\right) d t d s, \int_{B} G_{2}\left(t, s, w_{2}(t, s)\right) d s d t\right)
\end{aligned}
$$

for $\left(x, y, w_{0}, w_{1}, w_{2}\right) \in E \times C(B, \mathbb{R})^{3}$.

We construct a wide class of implicit and explicit difference schemes for the hyperbolic problem (1),(2). A general theory of convergence of such schemes for hyperbolic problems without partial derivatives on the righthand side of (1) was developed in [8]. Explicit difference schemes for (1), (2) were also considered in [10].

Difference methods for nonlinear parabolic functional differential problems were studied in [7], [9], [11]-[13]. Those investigations focused on finding a stable difference approximation which satisfies consistency conditions with respect to the original problem. Stability of corresponding nonlinear functional difference equations was proved by using difference inequalities or simple theorems on linear recurrent inequalities.

Difference methods for first order functional differential equations with initial or initial-boundary conditions were studied in [1], [4], the latter paper 
with rich bibliographical information. Convergence of these methods was proved by means of functional difference inequalities and a comparative method. We also refer the reader to [16] for a survey on difference methods for ordinary differential functional equations.

In this paper we construct difference schemes for (1), (2) under the assumption that $f$ is Lipschitzean with respect to the last two variables. In the case of implicit schemes we also assume that $f$ is Lipschitzean with respect to the third variable while for explicit schemes we only assume that $f$ satisfies a nonlinear estimate of the Perron type. In the latter case we also prove the existence and uniqueness for implicit functional difference problems that arise in discretization of (1), (2).

Existence results for the Darboux problem for functional differential problems can be found in [2], [3], [14].

2. The difference problem. For any two sets $X$ and $Y$, we denote by $\mathcal{F}[X, Y]$ the set of all functions from $X$ to $Y$.

We construct a mesh in $E^{0} \cup E$ in the following way. Let $h \in(0, a]$ and $k \in(0, b]$ denote the steps of the mesh with respect to $x$ and $y$. Write

$$
x_{i}=i h, \quad y_{j}=j k, \quad x_{i+1 / 2}=i h+h / 2, \quad y_{j+1 / 2}=j k+k / 2, \quad i, j \in \mathbb{Z} .
$$

We denote by $I_{0}$ the set of all $(h, k) \in(0, a] \times(0, b]$ for which there exist $M_{0}, N_{0} \in \mathbb{N}$ such that $M_{0} h=a_{0}, N_{0} k=b_{0}$ and such that $k / c \leq h \leq k c$ for a fixed constant $c \geq 1$. We assume that $I_{0}$ is nonempty and that there exists a sequence $\left\{\left(h_{n}, k_{n}\right)\right\} \subset I_{0}$ such that $\lim _{n \rightarrow \infty}\left(h_{n}, k_{n}\right)=(0,0)$. For $(h, k) \in I_{0}$ put $Z_{h k}=\left\{\left(x_{i}, y_{j}\right): i, j \in \mathbb{Z}\right\}$ and

$$
E_{h k}^{0}=Z_{h k} \cap E^{0}, \quad E_{h k}=Z_{h k} \cap E, \quad \bar{E}_{h k}=Z_{h k} \cap \bar{E} .
$$

There exist $M, N \in \mathbb{N}$ such that $M h \leq a<(M+1) h$ and $N k \leq b<(N+1) k$. Then

Furthermore, let

$$
E_{h k}=\left\{\left(x_{i}, y_{j}\right): 1 \leq i \leq M, 1 \leq j \leq N\right\} .
$$

$$
\begin{aligned}
& E_{h k}^{1}=\left\{\left(x_{i}, y_{j}\right): 1 \leq i \leq M-K, 1 \leq j \leq N\right\}, \\
& E_{h k}^{2}=\left\{\left(x_{i}, y_{j}\right): 1 \leq i \leq M, 1 \leq j \leq N-K\right\},
\end{aligned}
$$

where $K=0$ or $K=1$. With the same $K$ we write

$$
\begin{aligned}
& B_{h k}=\left\{\left(x_{i}, y_{j}\right):-M_{0} \leq i \leq K,-N_{0} \leq j \leq K\right\}, \\
& B_{h k}^{1}=\left\{\left(x_{i}, y_{j}\right):-M_{0} \leq i \leq 0,-N_{0} \leq j \leq K\right\}, \\
& B_{h k}^{2}=\left\{\left(x_{i}, y_{j}\right):-M_{0} \leq i \leq K,-N_{0} \leq j \leq 0\right\} .
\end{aligned}
$$

Given $\omega \in \mathcal{F}\left[E_{h k}^{0} \cup E_{h k}, \mathbb{R}\right]$ and $\left(x_{i}, y_{j}\right)$ we write $\omega^{(i, j)}$ instead of $\omega\left(x_{i}, y_{j}\right)$ for simplicity, and we use the same convention for $\mathcal{F}\left[E_{h k}^{0} \cup E_{h k}^{1}, \mathbb{R}\right]$ and $\mathcal{F}\left[E_{h k}^{0} \cup E_{h k}^{2}, \mathbb{R}\right]$. 
Now, we define a discrete version of the operator $(x, y) \mapsto \omega_{(x, y)}$. If $\omega: E_{h k}^{0} \cup E_{h k} \rightarrow \mathbb{R}$ and $0 \leq i \leq M-1,0 \leq j \leq N-1$ then we define $\omega_{[i, j]}: B_{h k} \rightarrow \mathbb{R}$ by

$$
\omega_{[i, j]}(t, s)=\omega\left(x_{i}+t, y_{j}+s\right), \quad(t, s) \in B_{h k} .
$$

If $K=1$ we analogously define $\omega_{[i, j]}$ on $B_{h k}^{1}$ and $B_{h k}^{2}$ for $\omega: E_{h k}^{0} \cup E_{h k}^{1} \rightarrow \mathbb{R}$ or $\omega: E_{h k}^{0} \cup E_{h k}^{2} \rightarrow \mathbb{R}$.

We define the following difference operators:

$$
\begin{aligned}
\delta \omega^{(i, j)} & =\frac{1}{h k}\left[\omega^{(i+1, j+1)}-\omega^{(i+1, j)}-\omega^{(i, j+1)}+\omega^{(i, j)}\right], \\
\delta_{1} \omega^{(i, j)} & =\frac{1}{h}\left[\omega^{(i+1, j)}-\omega^{(i, j)}\right], \quad \delta_{2} \omega^{(i, j)}=\frac{1}{k}\left[\omega^{(i, j+1)}-\omega^{(i, j)}\right] .
\end{aligned}
$$

Let $f_{h k}: \bar{E}_{h k} \times \mathcal{F}\left[B_{h k}, \mathbb{R}\right] \times \mathcal{F}\left[B_{h k}^{1}, \mathbb{R}\right] \times \mathcal{F}\left[B_{h k}^{2}, \mathbb{R}\right] \rightarrow \mathbb{R}$ and $\phi_{h k}, \alpha_{h k}, \beta_{h k}:$ $E_{h k}^{0} \rightarrow \mathbb{R}$ be given functions. We will approximate solutions of problem $(1),(2)$ by solutions of the following system of functional difference equations:

$$
\begin{array}{clrl}
\delta z^{(i, j)} & =f_{h k}\left(x_{i}, y_{j}, z_{[i, j]}, u_{[i, j]}, v_{[i, j]}\right), & \left(x_{i}, y_{j}\right) & \in E_{h k}-\{(h, k)\}, \\
\delta_{2} u^{(i, j)} & =f_{h k}\left(x_{i}, y_{j}, z_{[i, j]}, u_{[i, j]}, v_{[i, j]}\right), & \left(x_{i}, y_{j}\right) \in E_{h k}^{1}-\{(k, 0)\}, \\
\delta_{1} v^{(i, j)}=f_{h k}\left(x_{i}, y_{j}, z_{[i, j]}, u_{[i, j]}, v_{[i, j]}\right), & \left(x_{i}, y_{j}\right) \in E_{h k}-\{(0, h)\},
\end{array}
$$

where "-" denotes the algebraic difference of sets, with initial conditions

$$
z=\phi_{h k}, \quad u=\alpha_{h k}, \quad v=\beta_{h k}, \quad \text { on } E_{h k}^{0} .
$$

More precisely a solution $w$ of (1),(2) will be approximated by $z$ while the derivatives $D_{x} w, D_{y} w$ by $u, v$, respectively.

REMARK 1. If $K=1$ then problem (5), (6) becomes an implicit difference method. More precisely, for fixed $(i, j)$ the right-hand side of (5) depends not only on $z^{(\mu, \nu)}, u^{(\mu, \nu)}, v^{(\mu, \nu)}$ for $\mu \leq i, \nu \leq j$, but also on $z^{(i+1, j+1)}$, $z^{(i+1, j)}, z^{(i, j+1)}, u^{(i, j+1)}$ and $v^{(i+1, j)}$. Note that in this method we do not compute the values of $u^{(i, j)}$ for $i=M$ and the values of $v^{(i, j)}$ for $j=N$. If $K=0$ then we have an explicit method and problem (5), (6) represents a simple functional difference system of the Volterra type which obviously has exactly one solution.

In the case $K=1$ the existence theorem for the functional difference problem (5), (6) is proved by the use of the comparison method. This method consists in associating with the operator $f_{h k}$ another operator $\sigma_{h k}$ and investigating a suitable comparison equation. If the latter equation has adequate properties, then problem (5), (6) has exactly one solution which is the limit of the sequence of successive approximations. 
The general idea of the method used here is given in the fundamental paper of Ważewski [15]. This method has been used under various assumptions in many papers, for initial or initial-boundary value problems for partial or ordinary, differential and functional differential equations [3], [5], [6], [14].

Define the following discrete operators:

$$
\begin{array}{ll}
\mathcal{V}_{0} f_{h k}[z, u, v]^{(i, j)}=h k \sum_{\mu=0}^{i-1} \sum_{\nu=0}^{j-1} f_{h k}[z, u, v]^{(\mu, \nu)} & \text { on } E_{h k}, \\
\mathcal{V}_{1} f_{h k}[z, u, v]^{(i, j)}=k \sum_{\nu=0}^{j-1} f_{h k}[z, u, v]^{(i, \nu)} & \\
\mathcal{V}_{2} f_{h k}[z, u, v]^{(i, j)}=h \sum_{\mu=0}^{i-1} f_{h k}[z, u, v]^{(\mu, j)} & \text { on } E_{h k}^{1},
\end{array}
$$

where $f_{h k}[z, u, v]^{(i, j)}=f_{h k}\left(x_{i}, y_{j}, z_{[i, j]}, u_{[i, j]}, v_{[i, j]}\right)$.

Lemma 1. Problem (5), (6) is equivalent to

$$
\begin{array}{cr}
z^{(i, j)}=\mathcal{V}_{0} f_{h k}[z, u, v]^{(i, j)}+\phi_{h k}^{(i, 0)}+\phi_{h k}^{(0, j)}-\phi_{h k}^{(0,0)} & \text { on } E_{h k}, \\
u^{(i, j)}=\mathcal{V}_{1} f_{h k}[z, u, v]^{(i, j)}+\alpha_{h k}^{(i, 0)} & \text { on } E_{h k}^{1}, \\
v^{(i, j)}=\mathcal{V}_{2} f_{h k}[z, u, v]^{(i, j)}+\beta_{h k}^{(0, j)} & \text { on } E_{h k}^{2}, \\
z=\phi_{h k}, \quad u=\alpha_{h k}, \quad v=\beta_{h k}, & \text { on } E_{h k}^{0} .
\end{array}
$$

We omit the proof of this lemma.

REMARK 2. The proposed difference method is generated by the method of proving the existence of solutions of the Darboux problem in which (1), (2) is transformed into a system of integral equations

$$
\begin{aligned}
& z(x, y)=\int_{0}^{x} \int_{0}^{y} f\left(s, t, z_{(s, t)}, u_{(s, t)}, v_{(s, t)}\right) d s d t+\phi(x, 0)+\phi(0, y)-\phi(0,0), \\
& u(x, y)=\int_{0}^{y} f\left(x, t, z_{(x, t)}, u_{(x, t)}, v_{(x, t)}\right) d t+D_{x} \phi(x, 0), \\
& v(x, y)=\int_{0}^{x} f\left(s, y, z_{(s, y)}, u_{(s, y)}, v_{(s, y)}\right) d s+D_{y} \phi(0, y), \quad \text { on } E, \\
& z=\phi, \quad u=D_{x} \phi, \quad v=D_{y} \phi, \quad \text { on } E^{0},
\end{aligned}
$$

that corresponds to $(7),(8)$. 
In the sequel we denote by $\Xi$ the set of all triples $(z, u, v)$ where $z: E_{h k}^{0} \cup$ $E_{h k} \rightarrow \mathbb{R}, u: E_{h k}^{0} \cup E_{h k}^{1} \rightarrow \mathbb{R}, v: E_{h k}^{0} \cup E_{h k}^{2} \rightarrow \mathbb{R}$, and by $\Xi_{+}$the analogous set with the functions taking values in $\mathbb{R}_{+}$. If $(z, u, v),(\bar{z}, \bar{u}, \bar{v}) \in \Xi$, and

$$
z \leq \bar{z} \text { on } E_{h k}, \quad u \leq \bar{u} \text { on } E_{h k}^{1}, \quad v \leq \bar{v} \text { on } E_{h k}^{2},
$$

then we write $(z, u, v) \leq(\bar{z}, \bar{u}, \bar{v})$ on $\left(E_{h k}, E_{h k}^{1}, E_{h k}^{2}\right)$ for simplicity. If all these inequalities hold on the same set $E_{h k}^{0}$ then we use the usual notation $(z, u, v) \leq(\bar{z}, \bar{u}, \bar{v})$ on $E_{h k}^{0}$. Analogously we define the relation $(z, u, v)=$ $(\bar{z}, \bar{u}, \bar{v})$ on $\left(E_{h k}, E_{h k}^{1}, E_{h k}^{2}\right)$.

For $(z, u, v) \in \Xi$ define

$$
\mathcal{V} f_{h k}[z, u, v]=\left(\mathcal{V}_{0} f_{h k}[z, u, v], \mathcal{V}_{1} f_{h k}[z, u, v], \mathcal{V}_{2} f_{h k}[z, u, v]\right)
$$

Then we may write problem (7), (8) in a simple form

$$
\begin{aligned}
& (z, u, v)=\mathcal{V} f_{h k}[z, u, v]+\mathcal{T}\left(\phi_{h k}, \alpha_{h k}, \beta_{h k}\right) \quad \text { on }\left(E_{h k}, E_{h k}^{1}, E_{h k}^{2}\right), \\
& (z, u, v)=\left(\phi_{h k}, \alpha_{h k}, \beta_{h k}\right) \quad \text { on } E_{h k}^{0},
\end{aligned}
$$

where $\mathcal{T}$ is defined by

$$
\mathcal{T}\left(\phi_{h k}, \alpha_{h k}, \beta_{h k}\right)^{(i, j)}=\left(\phi_{h k}^{(i, 0)}+\phi_{h k}^{(0, j)}-\phi_{h k}^{(0,0)}, \alpha_{h k}^{(i, 0)}, \beta_{h k}^{(0, j)}\right) .
$$

For any $\omega_{h k}: E_{h k}^{0} \rightarrow \mathbb{R}$ let $\widetilde{\omega}_{h k}: E_{h k}^{0} \cup E_{h k} \rightarrow \mathbb{R}$ be defined by

$$
\widetilde{\omega}_{h k}(x, y)= \begin{cases}\omega_{h k}(x, y) & \text { for }(x, y) \in E_{h k}^{0}, \\ 0 & \text { for }(x, y) \in E_{h k},\end{cases}
$$

and analogously define $\widetilde{\omega}_{h k}$ on $E_{h k}^{0} \cup E_{h k}^{1}$ or $E_{h k}^{0} \cup E_{h k}^{2}$.

We define the sequence $\left\{\left(z_{n}, u_{n}, v_{n}\right)\right\} \subset \Xi$ in the following way:

(i) $\left(z_{0}, u_{0}, v_{0}\right)=\left(\widetilde{\phi}_{h k}, \widetilde{\alpha}_{h k}, \widetilde{\beta}_{h k}\right)$ on $\left(E_{h k}^{0} \cup E_{h k}, E_{h k}^{0} \cup E_{h k}^{1}, E_{h k}^{0} \cup E_{h k}^{2}\right)$;

(ii) if $\left(z_{n}, u_{n}, v_{n}\right) \in \Xi$ is already defined then

$$
\left(z_{n+1}, u_{n+1}, v_{n+1}\right)=\mathcal{V} f_{h k}\left[z_{n}, u_{n}, v_{n}\right]+\mathcal{T}\left(\phi_{h k}, \alpha_{h k}, \beta_{h k}\right)
$$

on $\left(E_{h k}, E_{h k}^{1}, E_{h k}^{2}\right)$, and

$$
\left(z_{n+1}, u_{n+1}, v_{n+1}\right)=\left(\phi_{h k}, \alpha_{h k}, \beta_{h k}\right) \quad \text { on } E_{h k}^{0} .
$$

We prove that under suitable assumptions on $f_{h k}$, the sequence $\left\{\left(z_{n}, u_{n}, v_{n}\right)\right\}$ converges to the unique solution of problem (7), (8).

For $\omega \in \mathcal{F}\left[B_{h k}, \mathbb{R}\right]$ we define a norm $|\omega|_{h k}$ by

$$
|\omega|_{h k}=\max \left\{|\omega(x, y)|:(x, y) \in B_{h k}\right\} .
$$

We let $\theta_{h k}$ be the zero function in $\mathcal{F}\left[B_{h k}, \mathbb{R}\right]$. We use the same notation for functions defined on $B_{h k}^{1}$ or $B_{h k}^{2}$.

Finally, if $\omega, \bar{\omega}: E_{h k}^{0} \rightarrow \mathbb{R}$ then $|\omega|: E_{h k} \rightarrow \mathbb{R}$ is defined by

$$
|\omega|^{(i, j)}=\left|\omega^{(i, j)}\right| \quad \text { on } E_{h k}^{0},
$$

and the relation $\omega \leq \bar{\omega}$ means that $\omega^{(i, j)} \leq \bar{\omega}^{(i, j)}$ on $E_{h k}^{0}$. 
Assumption $\mathrm{H}_{1}$. Suppose that

(i) for each fixed $(x, y) \in \bar{E}_{h k}$ the function $\sigma_{h k}(x, y, \cdot, \cdot, \cdot): \mathcal{F}\left[B_{h k}, \mathbb{R}\right] \times$ $\mathcal{F}\left[B_{h k}^{1}, \mathbb{R}\right] \times \mathcal{F}\left[B_{h k}^{2}, \mathbb{R}\right] \rightarrow \mathbb{R}_{+}$is continuous and nondecreasing with respect to all its arguments, and $\sigma_{h k}\left(x, y, \theta_{h k}, \theta_{h k}, \theta_{h k}\right)=0$ for $(x, y) \in \bar{E}_{h k}$;

(ii) for all $(x, y) \in \bar{E}_{h k}, w, \bar{w} \in \mathcal{F}\left[B_{h k}, \mathbb{R}\right], w_{1}, \bar{w}_{1} \in \mathcal{F}\left[B_{h k}^{1}, \mathbb{R}\right], w_{2}, \bar{w}_{2} \in$ $\mathcal{F}\left[B_{h k}^{2}, \mathbb{R}\right]$ we have

$$
\begin{aligned}
& \left|f_{h k}\left(x, y, w, w_{1}, w_{2}\right)-f_{h k}\left(x, y, \bar{w}, \bar{w}_{1}, \bar{w}_{2}\right)\right| \\
& \leq \sigma_{h k}\left(x, y,|w-\bar{w}|_{h k},\left|w_{1}-\bar{w}_{1}\right|_{h k},\left|w_{2}-\bar{w}_{2}\right|_{h k}\right) ;
\end{aligned}
$$

(iii) there exists a solution $\left(g_{h k}, p_{h k}, q_{h k}\right) \in \Xi$ of the problem

$$
\begin{aligned}
& (z, u, v) \geq \mathcal{V} \sigma_{h k}[z, u, v]+\left(\eta_{h k}, \xi_{h k}, \lambda_{h k}\right) \quad \text { on }\left(E_{h k}, E_{h k}^{1}, E_{h k}^{2}\right), \\
& (z, u, v)=\left(\left|\phi_{h k}\right|,\left|\alpha_{h k}\right|,\left|\beta_{h k}\right|\right) \quad \text { on } E_{h k}^{0},
\end{aligned}
$$

where $\left(\eta_{h k}, \xi_{h k}, \lambda_{h k}\right)$ is such that

$$
\begin{aligned}
& \eta_{h k}^{(i, j)} \geq h k \sum_{\mu=0}^{i-1} \sum_{\nu=0}^{j-1}\left|f_{h k}\left[\theta_{h k}, \theta_{h k}, \theta_{h k}\right]^{(\mu, \nu)}\right|+\left|\phi_{h k}^{(i, 0)}\right|+\left|\phi_{h k}^{(0, j)}\right|+\left|\phi_{h k}^{(0,0)}\right| \\
& \xi_{h k}^{(i, j)} \geq k \sum_{\nu=0}^{j-1}\left|f_{h k}\left[\theta_{h k}, \theta_{h k}, \theta_{h k}\right]^{(i, \nu)}\right|+\left|\alpha_{h k}^{(i, 0)}\right| \\
& \lambda_{h k}^{(i, j)} \geq h \sum_{\mu=0}^{i-1}\left|f_{h k}\left[\theta_{h k}, \theta_{h k}, \theta_{h k}\right]^{(\mu, j)}\right|+\left|\beta_{h k}^{(0, j)}\right|
\end{aligned}
$$

on $E_{h k}, E_{h k}^{1}, E_{h k}^{2}$, respectively;

(iv) the function $(\bar{z}, \bar{u}, \bar{v})$, where $\bar{z}(x, y) \equiv 0, \bar{u}(x, y) \equiv 0, \bar{v}(x, y) \equiv 0$, is a unique solution of the problem

$$
\begin{aligned}
& (z, u, v)=\mathcal{V} \sigma_{h k}[z, u, v] \quad \text { on }\left(E_{h k}, E_{h k}^{1}, E_{h k}^{2}\right), \\
& (z, u, v)=0 \quad \text { on } E_{h k}^{0},
\end{aligned}
$$

in the class of all $(z, u, v) \in \Xi$ such that

$$
0 \leq(z, u, v) \leq\left(g_{h k}, p_{h k}, q_{h k}\right) \quad \text { on }\left(E_{h k}, E_{h k}^{1}, E_{h k}^{2}\right) .
$$

THEOREM 2. If Assumption $\mathrm{H}_{1}$ is satisfied then there is a solution $(\bar{z}, \bar{u}, \bar{v})$ $\in \Xi$ of problem (5),(6), and it is unique in the class of all $(z, u, v) \in \Xi$ such that

$$
(|z|,|u|,|v|) \leq\left(g_{h k}, p_{h k}, q_{h k}\right) \quad \text { on }\left(E_{h k}, E_{h k}^{1}, E_{h k}^{2}\right) .
$$

Proof. We consider the sequence $\left\{\left(g_{n}, p_{n}, q_{n}\right)\right\} \subset \Xi$ defined in the following way:

(i) $g_{0}=g_{h k}, p_{0}=p_{h k}, q_{0}=q_{h k}$; 
(ii) if $\left(g_{n}, p_{n}, q_{n}\right) \in \Xi$ is already defined then

$$
\begin{array}{lll}
\left(g_{n+1}, p_{n+1}, q_{n+1}\right)=\mathcal{V} \sigma_{h k}\left[g_{n}, p_{n}, q_{n}\right] & & \text { on }\left(E_{h k}, E_{h k}^{1}, E_{h k}^{2}\right), \\
\left(g_{n+1}, p_{n+1}, q_{n+1}\right)=0 & & \text { on } E_{h k}^{0} .
\end{array}
$$

We prove that on $\left(E_{h k}, E_{h k}^{1}, E_{h k}^{2}\right)$ we have

$$
\begin{gathered}
\left(g_{n+1}, p_{n+1}, q_{n+1}\right) \leq\left(g_{n}, p_{n}, q_{n}\right), \\
\lim _{n \rightarrow \infty}\left(g_{n}, p_{n}, q_{n}\right)=0, \\
\left(\left|z_{n}\right|,\left|u_{n}\right|,\left|v_{n}\right|\right) \leq\left(g_{h k}, p_{h k}, q_{h k}\right), \\
\left(\left|z_{n+1}-z_{n}\right|,\left|u_{n+1}-u_{n}\right|,\left|v_{n+1}-v_{n}\right|\right) \leq\left(g_{n}, p_{n}, q_{n}\right) .
\end{gathered}
$$

It follows from $(12)$ that $\left(g_{1}, p_{1}, q_{1}\right) \leq\left(g_{0}, p_{0}, q_{0}\right)$ on $\left(E_{h k}, E_{h k}^{1}, E_{h k}^{2}\right)$. If for fixed $n \in \mathbb{N}$ we have $\left(g_{n}, p_{n}, q_{n}\right) \leq\left(g_{n-1}, p_{n-1}, q_{n-1}\right)$, then from the monotonicity of $\sigma_{h k}$ with respect to the function variables we get $\left(g_{n+1}, p_{n+1}\right.$, $\left.q_{n+1}\right) \leq\left(g_{n}, p_{n}, q_{n}\right)$ and (19) follows by induction on $n$. Since we also have $\left(g_{n}, p_{n}, q_{n}\right) \geq 0$ on $\left(E_{h k}, E_{h k}^{1}, E_{h k}^{2}\right)$, the limit $(\bar{g}, \bar{p}, \bar{q})=\lim _{n \rightarrow \infty}\left(g_{n}, p_{n}, q_{n}\right)$ exists, and it follows from (17) and (18) that $(\bar{g}, \bar{p}, \bar{q}) \in \Xi$ is a solution of problem (15), (16), which implies $(\bar{g}, \bar{p}, \bar{q})=0$.

Note that inequality (21) is obvious for $n=0$. If we assume that $\left(\left|z_{n}\right|,\left|u_{n}\right|,\left|v_{n}\right|\right) \leq\left(g_{h k}, p_{h k}, q_{h k}\right)$, then from (11) and (14) and from the monotonicity of $\sigma_{h k}$ we get

$$
\begin{aligned}
\left|z_{n+1}^{(i, j)}\right| \leq & h k \sum_{\mu=0}^{i-1} \sum_{\nu=0}^{j-1}\left|f_{h k}\left[z_{n}, u_{n}, v_{n}\right]^{(\mu, \nu)}-f_{h k}\left[\theta_{h k}, \theta_{h k}, \theta_{h k}\right]^{(\mu, \nu)}\right| \\
& +h k \sum_{\mu=0}^{i-1} \sum_{\nu=0}^{j-1}\left|f_{h k}\left[\theta_{h k}, \theta_{h k}, \theta_{h k}\right]^{(\mu, \nu)}\right|+\left|\phi_{h k}^{(i, 0)}\right|+\left|\phi_{h k}^{(0, j)}\right|+\left|\phi_{h k}^{(0,0)}\right| \\
\leq & h k \sum_{\mu=0}^{i-1} \sum_{\nu=0}^{j-1} \sigma_{h k}\left(x_{\mu}, y_{\nu},\left(g_{h k}\right)_{[\mu, \nu]},\left(p_{h k}\right)_{[\mu, \nu]},\left(q_{h k}\right)_{[\mu, \nu]}\right)+\eta_{h k}^{(i, j)} \\
\leq & g_{h k}^{(i, j)}
\end{aligned}
$$

on $E_{h k}$, and analogously

$$
\begin{aligned}
\left|u_{n+1}^{(i, j)}\right| & \leq k \sum_{\nu=0}^{j-1} \sigma_{h k}\left(x_{i}, y_{\nu},\left(g_{h k}\right)_{[i, \nu]},\left(p_{h k}\right)_{[i, \nu]},\left(q_{h k}\right)_{[i, \nu]}\right)+\xi_{h k}^{(i, j)} \leq p_{h k}^{(i, j)}, \\
\left|v_{n+1}^{(i, j)}\right| & \leq h \sum_{\mu=0}^{i-1} \sigma_{h k}\left(x_{\mu}, y_{j},\left(g_{h k}\right)_{[\mu, j]},\left(p_{h k}\right)_{[\mu, j]},\left(q_{h k}\right)_{[\mu, j]}\right)+\lambda_{h k}^{(i, j)} \leq q_{h k}^{(i, j)},
\end{aligned}
$$

on $E_{h k}^{1}, E_{h k}^{2}$, respectively. Thus (21) follows by induction for all $n$, and consequently (22) holds for $n=0$. If we assume that $\left(\left|z_{n+r}-z_{n}\right|,\left|u_{n+r}-u_{n}\right|\right.$, $\left.\left|v_{n+r}-v_{n}\right|\right) \leq\left(g_{n}, p_{n}, q_{n}\right)$, then from (11) and from the monotonicity of $\sigma_{h k}$ 
we get

$$
\begin{aligned}
& \left|z_{n+r+1}^{(i, j)}-z_{n+1}^{(i, j)}\right| \\
& \quad \leq h k \sum_{\mu=0}^{i-1} \sum_{\nu=0}^{j-1}\left|f_{h k}\left[z_{n+r}, u_{n+r}, v_{n+r}\right]^{(\mu, \nu)}-f_{h k}\left[z_{n}, u_{n}, v_{n}\right]^{(\mu, \nu)}\right| \\
& \quad \leq h k \sum_{\mu=0}^{i-1} \sum_{\nu=0}^{j-1} \sigma_{h k}\left(x_{\mu}, y_{\nu},\left|\left(z_{n+r}-z_{n}\right)_{[\mu, \nu]}\right|_{h k},\right. \\
& \left.\quad \leq h k \sum_{\mu=0}^{i-1} \sum_{\nu=0}^{j-1} \sigma_{h k}\left(x_{\mu}, y_{\nu},\left(g_{n}\right)_{[\mu, \nu]},\left(p_{n}\right)_{[\mu, \nu]},\left(q_{n}\right)_{[\mu, \nu]}\right)=g_{n+1}^{(i, j)} \quad \text { on } E_{h k},\left|\left(v_{n+r}-v_{n}\right)_{[\mu, \nu]}\right|_{h k}\right)
\end{aligned}
$$

and analogously

$$
\left|u_{n+r+1}^{(i, j)}-u_{n+1}^{(i, j)}\right| \leq p_{n+1}^{(i, j)} \quad \text { on } E_{h k}^{1}, \quad\left|v_{n+r+1}^{(i, j)}-v_{n+1}^{(i, j)}\right| \leq q_{n+1}^{(i, j)} \quad \text { on } E_{h k}^{2},
$$

from which (22) follows by induction again.

It follows from $(20)$ and $(22)$ that $(\bar{z}, \bar{u}, \bar{v})=\lim _{n \rightarrow \infty}\left(z_{n}, u_{n}, v_{n}\right)$ exists. By (9) and (10) we see that $(\bar{z}, \bar{u}, \bar{v}) \in \Xi$ is a solution of problem (7), (8). Suppose that we have another solution $(\widehat{z}, \widehat{u}, \widehat{v}) \in \Xi$ of $(7),(8)$ such that $(|\widehat{z}|,|\widehat{u}|,|\widehat{v}|) \leq\left(g_{h k}, p_{h k}, q_{h k}\right)$ on $\left(E_{h k}, E_{h k}^{1}, E_{h k}^{2}\right)$. Then as in the proof of (22) we get $\left(\left|\widehat{z}-z_{n}\right|,\left|\widehat{u}-u_{n}\right|,\left|\widehat{v}-v_{n}\right|\right) \leq\left(g_{n}, p_{n}, q_{n}\right)$, from which, by $(20)$, it follows that $(\widehat{z}, \widehat{u}, \widehat{v})=(\bar{z}, \bar{u}, \bar{v})$.

REMARK 3. Theorem 2 gives uniqueness of solutions of problem (5), (6) only in the class of $(z, u, v) \in \Xi$ such that

$$
(|z|,|u|,|v|) \leq\left(g_{h k}, p_{h k}, q_{h k}\right) \quad \text { on }\left(E_{h k}, E_{h k}^{1}, E_{h k}^{2}\right)
$$

We may also get global uniqueness provided that Assumption $\mathrm{H}_{1}$ is satisfied and that $(\bar{z}, \bar{u}, \bar{v}) \equiv(0,0,0)$ is a unique solution of the problem

$$
\begin{aligned}
& (z, u, v) \leq \mathcal{V} \sigma_{h k}[z, u, v] \quad \text { on }\left(E_{h k}, E_{h k}^{1}, E_{h k}^{2}\right), \\
& (z, u, v)=0 \quad \text { on } E_{h k}^{0} .
\end{aligned}
$$

3. Convergence of explicit difference methods. The next lemma concerns functional difference inequalities generated by monotone operators of the Volterra type.

Lemma 3. Suppose that $K=0$ and

(i) $G_{h k}: \bar{E}_{h k} \times \mathcal{F}\left[B_{h k}, \mathbb{R}\right]^{3} \rightarrow \mathbb{R}$ is nondecreasing with respect to the last three variables; 
(ii) $z, u, v, \bar{z}, \bar{u}, \bar{v}: E_{h k}^{0} \cup E_{h k} \rightarrow \mathbb{R}$ satisfy the inequalities

$$
\begin{aligned}
(z, u, v)-\mathcal{V} G_{h k}[z, u, v] & \leq(\bar{z}, \bar{u}, \bar{v})-\mathcal{V} G_{h k}[\bar{z}, \bar{u}, \bar{v}] \quad \text { on } E_{h k} \\
(z, u, v) & \leq(\bar{z}, \bar{u}, \bar{v}) \quad \text { on } E_{h k}^{0} .
\end{aligned}
$$

Then

$$
(z, u, v) \leq(\bar{z}, \bar{u}, \bar{v}) \quad \text { on } E_{h k} .
$$

The proof is by induction and we omit it.

To state the next assumptions we denote by $\Theta$ the class of all functions $\alpha: I_{0} \rightarrow \mathbb{R}_{+}$such that $\lim _{(h, k) \rightarrow(0,0)} \alpha(h, k)=0$.

Assumption $\mathrm{H}_{2}$. Suppose that $K=0$ and

(i) conditions (i), (ii) and (iv) of Assumption $\mathrm{H}_{1}$ are satisfied;

(ii) the solution $(\bar{z}, \bar{u}, \bar{v}) \in \Xi_{+}$, where $\bar{z}(x, y) \equiv 0, \bar{u}(x, y) \equiv 0, \bar{v}(x, y)$ $\equiv 0$, of the problem

$$
\begin{array}{cl}
\delta z^{(i, j)}=\sigma_{h k}[z, u, v]^{(i, j)}, & \left(x_{i}, y_{j}\right) \in E_{h k}-\{(h, k)\}, \\
\delta_{2} u^{(i, j)}=\sigma_{h k}[z, u, v]^{(i, j)}, \quad\left(x_{i}, y_{j}\right) \in E_{h k}-\{(0, k)\}, \\
\delta_{1} v^{(i, j)}=\sigma_{h k}[z, u, v]^{(i, j)}, \quad\left(x_{i}, y_{j}\right) \in E_{h k}-\{(h, 0)\}, \\
z^{(i, j)}=u^{(i, j)}=v^{(i, j)}=0, \quad \text { on } E_{h k}^{0},
\end{array}
$$

is stable in the following sense: if $\left(\widetilde{z}_{h k}, \widetilde{u}_{h k}, \widetilde{v}_{h k}\right) \in \Xi_{+}$is a solution of the problem

$$
\begin{array}{cc}
\delta z^{(i, j)}=\sigma_{h k}[z, u, v]^{(i, j)}+\alpha_{0}(h, k), & \left(x_{i}, y_{j}\right) \in E_{h k}-\{(h, k)\}, \\
\delta_{2} u^{(i, j)}=\sigma_{h k}[z, u, v]^{(i, j)}+\alpha_{1}(h, k), & \left(x_{i}, y_{j}\right) \in E_{h k}-\{(0, k)\}, \\
\delta_{1} v^{(i, j)}=\sigma_{h k}[z, u, v]^{(i, j)}+\alpha_{2}(h, k), & \left(x_{i}, y_{j}\right) \in E_{h k}-\{(h, 0)\}, \\
z^{(i, j)}=\varepsilon_{0}(h, k), \quad u^{(i, j)}=\varepsilon_{1}(h, k), & v^{(i, j)}=\varepsilon_{2}(h, k), \quad \text { on } E_{h k}^{0},
\end{array}
$$

where $\alpha_{i}, \varepsilon_{i} \in \Theta, i=0,1,2$, then there are $\beta_{i} \in \Theta, i=0,1,2$, such that

$$
\widetilde{z}_{h k}^{(i, j)} \leq \beta_{0}(h, k), \quad \widetilde{u}_{h k}^{(i, j)} \leq \beta_{1}(h, k), \quad \widetilde{v}_{h k}^{(i, j)} \leq \beta_{2}(h, k), \quad \text { on } E_{h k} .
$$

An example of a class of difference problems satisfying Assumption $\mathrm{H}_{2}$ is given in Theorem 5 .

TheOREm 4. Suppose that Assumption $\mathrm{H}_{2}$ is satisfied and

(i) $\left(z_{h k}, u_{h k}, v_{h k}\right) \in \Xi$ is a solution of problem (5), (6) and there are $\alpha_{i} \in \Theta, i=0,1,2$, such that

$$
\begin{gathered}
\left|\phi^{(i, j)}-\phi_{h k}^{(i, j)}\right| \leq \varepsilon_{0}(h, k), \quad\left|\left(D_{x} \phi\right)^{(i, j)}-\alpha_{h k}^{(i, j)}\right| \leq \varepsilon_{1}(h, k), \\
\left|\left(D_{y} \phi\right)^{(i, j)}-\beta_{h k}^{(i, j)}\right| \leq \varepsilon_{2}(h, k), \quad \text { on } E_{h k}^{0} ;
\end{gathered}
$$

(ii) $w: E^{0} \cup E \rightarrow \mathbb{R}$ is a solution of problem (1), (2) and $w$ is of class $C^{3}$ on $\bar{E}$; 
(iii) the compatibility condition

$$
\begin{aligned}
& \mid f_{h k}\left(x_{i}, y_{j},\left(w_{h k}\right)_{[i, j]},\left(D_{x} w_{h k}\right)_{[i, j]},\left(D_{y} w_{h k}\right)_{[i j]}\right) \\
& \quad-f\left(x_{i}, y_{j}, w_{\left(x_{i}, y_{j}\right)},\left(D_{x} w\right)_{\left(x_{i}, y_{j}\right)},\left(D_{y} w\right)_{\left(x_{i}, y_{j}\right)}\right) \mid \leq \widetilde{\alpha}(h, k)
\end{aligned}
$$

is satisfied on $\bar{E}_{h k}$ with $\widetilde{\alpha} \in \Theta$, where $w_{h k}, D_{x} w_{h k}, D_{y} w_{h k}$ are the restrictions of $w, D_{x} w, D_{y} w$, respectively, to $E_{h k}^{0} \cup E_{h k}$.

Under these assumptions there exist $\beta_{i} \in \Theta, i=0,1,2$, such that

$$
\begin{gathered}
\left|w^{(i, j)}-z_{h k}^{(i, j)}\right| \leq \beta_{0}(h, k), \quad\left|\left(D_{x} w\right)^{(i, j)}-u_{h k}^{(i, j)}\right| \leq \beta_{1}(h, k), \\
\left|\left(D_{y} w\right)^{(i, j)}-v_{h k}^{(i, j)}\right| \leq \beta_{2}(h, k), \quad \text { on } E_{h k} .
\end{gathered}
$$

Proof. Let $\eta_{h k}, \xi_{h k}, \lambda_{h k}$ be defined by

$$
\begin{aligned}
\delta w_{h k}^{(i, j)} & =f_{h k}\left[w_{h k}, D_{x} w_{h k}, D_{y} w_{h k}\right]^{(i, j)}+\eta_{h k}^{(i, j)}, \\
\delta_{2} D_{x} w_{h k}^{(i, j)} & =f_{h k}\left[w_{h k}, D_{x} w_{h k}, D_{y} w_{h k}\right]^{(i, j)}+\xi_{h k}^{(i, j)}, \\
\delta_{1} D_{y} w_{h k}^{(i, j)} & =f_{h k}\left[w_{h k}, D_{x} w_{h k}, D_{y} w_{h k}\right]^{(i, j)}+\lambda_{h k}^{(i, j)} .
\end{aligned}
$$

From the compatibility condition (26) and (ii) it follows that there are $\alpha_{i} \in$ $\Theta, i=0,1,2$, such that

$$
\left|\eta_{h k}^{(i, j)}\right| \leq \alpha_{0}(h, k), \quad\left|\xi_{h k}^{(i, j)}\right| \leq \alpha_{1}(h, k), \quad\left|\lambda_{h k}^{(i, j)}\right| \leq \alpha_{0}(h, k) .
$$

Let $\widetilde{z}_{h k}^{(i, j)}=\left|z_{h k}^{(i, j)}-w^{(i, j)}\right|, \widetilde{u}_{h k}^{(i, j)}=\left|u_{h k}^{(i, j)}-\left(D_{x} w\right)^{(i, j)}\right|, \widetilde{v}_{h k}^{(i, j)}=\mid v_{h k}^{(i, j)}-$ $\left(D_{y} w\right)^{(i, j)} \mid$. Then

$$
\begin{aligned}
\widetilde{z}_{h k}^{(i, j)} & \leq \mathcal{V}_{0} \sigma_{h k}\left[\widetilde{z}_{h k}, \widetilde{u}_{h k}, \widetilde{v}_{h k}\right]^{(i, j)}+h i k j \alpha_{0}(h, k), \\
\widetilde{u}_{h k}^{(i, j)} & \leq \mathcal{V}_{1} \sigma_{h k}\left[\widetilde{z}_{h k}, \widetilde{u}_{h k}, \widetilde{v}_{h k}\right]^{(i, j)}+k j \alpha_{1}(h, k), \\
\widetilde{v}_{h k}^{(i, j)} & \leq \mathcal{V}_{2} \sigma_{h k}\left[\widetilde{z}_{h k}, \widetilde{u}_{h k}, \widetilde{v}_{h k}\right]^{(i, j)}+h i \alpha_{2}(h, k), \quad \text { on } E_{h k}, \\
\widetilde{z}_{h k}^{(i, j)} & \leq \varepsilon_{0}(h, k), \quad \widetilde{u}_{h k}^{(i, j)} \leq \varepsilon_{1}(h, k), \quad \widetilde{v}_{h k}^{(i, j)} \leq \varepsilon_{2}(h, k), \quad \text { on } E_{h k}^{0} .
\end{aligned}
$$

Let $(\bar{z}, \bar{u}, \bar{v}) \in \Xi$ be a solution of the problem

$$
\begin{aligned}
& z^{(i, j)}=\mathcal{V}_{0} \sigma_{h k}[z, u, v]^{(i, j)}+a b \alpha_{0}(h, k), \\
& u^{(i, j)}=\mathcal{V}_{1} \sigma_{h k}[z, u, v]^{(i, j)}+b \alpha_{1}(h, k), \\
& v^{(i, j)}=\mathcal{V}_{2} \sigma_{h k}[z, u, v]^{(i, j)}+a \alpha_{2}(h, k), \quad \text { on } E_{h k}, \\
z^{(i, j)}= & \varepsilon_{0}(h, k), \quad u^{(i, j)}=\varepsilon_{1}(h, k), \quad v^{(i, j)}=\varepsilon_{2}(h, k), \quad \text { on } E_{h k}^{0} .
\end{aligned}
$$

Lemma 3 implies that

$$
\widetilde{z}_{h k}^{(i, j)} \leq \bar{z}^{(i, j)}, \quad \widetilde{u}_{h k}^{(i, j)} \leq \bar{u}^{(i, j)}, \quad \widetilde{v}_{h k}^{(i, j)} \leq \bar{v}^{(i, j)}, \quad \text { on } E_{h k} .
$$

The stability of problem (23), (24) completes the proof of Theorem 4. 
We consider the difference method

$$
\begin{aligned}
\delta z^{(i, j)} & =f\left(x_{i}, y_{j}, T_{h k} z_{[i, j]}, T_{h k} u_{[i, j]}, T_{h k} v_{[i, j]}\right), \\
\delta_{2} u^{(i, j)} & =f\left(x_{i}, y_{i}, T_{h k} z_{[i, j]}, T_{h k} u_{[i, j]}, T_{h k} v_{[i, j]}\right), \\
\delta_{1} v^{(i, j)} & =f\left(x_{i}, y_{j}, T_{h k} z_{[i, j]}, T_{h k} u_{[i, j]}, T_{h k} v_{[i, j]}\right),
\end{aligned}
$$

on $E_{h k}-\{(h, k)\}, E_{h k}-\{(0, k)\}, E_{h k}-\{(h, 0)\}$, respectively, and

$$
z=\phi_{h k}, \quad u=\alpha_{h k}, \quad v=\beta_{h k}, \quad \text { on } E_{h k}^{0},
$$

where $T_{h k}: \mathcal{F}\left[B_{h k}, \mathbb{R}\right] \rightarrow C(B, \mathbb{R})$ is an interpolating operator defined by

$$
\begin{aligned}
& T_{h k} w(t, s)=w^{(i+1, j+1)} \frac{t-x_{i}}{h} \frac{s-y_{j}}{k}+w^{(i, j+1)}\left[1-\frac{t-x_{i}}{h}\right] \frac{s-y_{j}}{k} \\
& +w^{(i+1, j)} \frac{t-x_{i}}{h}\left[1-\frac{s-y_{j}}{k}\right]+w^{(i, j)}\left[1-\frac{t-x_{i}}{h}\right]\left[1-\frac{s-y_{j}}{k}\right],
\end{aligned}
$$

for $(t, s) \in B$, where $i, j<0$ are integers such that $x_{i} \leq t \leq x_{i+1}$ and $y_{j} \leq s \leq y_{j+1}$.

The above problem may be considered as a generalization of the Euler method for ordinary functional differential equations.

Assumption $\mathrm{H}_{3}$. Suppose that $f: \bar{E} \times C(B, \mathbb{R})^{3} \rightarrow \mathbb{R}$ is continuous and

(i) there is a continuous and nondecreasing function $\sigma: \bar{E} \times \mathbb{R}_{+} \rightarrow \mathbb{R}_{+}$ such that $\sigma(x, y, 0)=0$ for $(x, y) \in \bar{E}$;

(ii) the function $\bar{\omega}(x, y)=0,(x, y) \in \bar{E}$, is a unique solution of the problem

$$
\begin{aligned}
D_{x y} z(x, y) & =\sigma(x, y, z(x, y))+L_{1} D_{x} z(x, y)+L_{2} D_{y} z(x, y), \quad(x, y) \in \bar{E}, \\
z(x, 0) & =0 \quad \text { for } x \in[0, a], \quad z(0, y)=0 \text { for } y \in[0, b],
\end{aligned}
$$

where $L_{1}, L_{2}$ are nonnegative constants;

(iii) the estimate

$$
\begin{aligned}
& \left|f\left(x, y, w, w_{1}, w_{2}\right)-f\left(x, y, \bar{w}, \bar{w}_{1}, \bar{w}_{2}\right)\right| \\
& \leq \sigma\left(x, y,\|w-\bar{w}\|_{B}\right)+L_{1}\left\|w_{1}-\bar{w}_{1}\right\|_{B}+L_{2}\left\|w_{2}-\bar{w}_{2}\right\|_{B}
\end{aligned}
$$

is satisfied on $\bar{E} \times C(B, \mathbb{R})^{3}$, where $\|\cdot\|_{B}$ denotes the supremum norm in $C(B, \mathbb{R})$.

Theorem 5. Suppose that $K=0$, Assumption $\mathrm{H}_{3}$ is satisfied and

(i) $\left(z_{h k}, u_{h k}, v_{h k}\right) \in \Xi$ is a solution of problem $(27),(28)$ and there are $\varepsilon_{i} \in \Theta, i=0,1,2$, such that (25) holds;

(ii) $w: E^{0} \cup \bar{E} \rightarrow \mathbb{R}$ is a solution of problem (1),(2) and $w$ is of class $C^{4}$ on $\bar{E}$. 
Then there are $\beta_{i} \in \Theta, i=0,1,2$, such that

$$
\begin{gathered}
\left|z_{h k}^{(i, j)}-w^{(i, j)}\right| \leq \beta_{0}(h, k), \quad\left|u_{h k}^{(i, j)}-\left(D_{x} w\right)^{(i, j)}\right| \leq \beta_{1}(h, k), \\
\left|v_{h k}^{(i, j)}-\left(D_{y} w\right)^{(i, j)}\right| \leq \beta_{2}(h, k), \quad \text { on } E_{h k} .
\end{gathered}
$$

Pro of. Since $K=0$ we have $B_{h k}=B_{h k}^{1}=B_{h k}^{2}$. Let

$$
f_{h k}\left(x, y, w, w_{1}, w_{2}\right)=f\left(x, y, T_{h k} w, T_{h k} w_{1}, T_{h k} w_{2}\right)
$$

for $\left(x, y, w, w_{1}, w_{2}\right) \in \bar{E}_{h k} \times \mathcal{F}\left[B_{h k}, \mathbb{R}\right]^{3}$. Then

$\left|f_{h k}\left(x, y, w, w_{1}, w_{2}\right)-f_{h k}\left(x, y, \bar{w}, \bar{w}_{1}, \bar{w}_{2}\right)\right|$

$\leq \sigma\left(x, y,\left\|T_{h k}(w-\bar{w})\right\|_{B}\right)+L_{1}\left\|T_{h k}\left(w_{1}-\bar{w}_{1}\right)\right\|_{B}+L_{2}\left\|T_{h k}\left(w_{2}-\bar{w}_{2}\right)\right\|_{B}$

$=\sigma\left(x, y,|w-\bar{w}|_{h k}\right)+L_{1}\left|w_{1}-\bar{w}_{1}\right|_{h k}+L_{2}\left|w_{2}-\bar{w}_{2}\right|_{h k}$

on $\bar{E}_{h k} \times \mathcal{F}\left[B_{h k}, \mathbb{R}\right]^{3}$. The operator $T_{h k}$ has the following property: if $w \in$ $\mathcal{F}(B, \mathbb{R})$ is of class $C^{3}$ then there is $\widetilde{C}>0$ such that

$$
\left\|T_{h k} w_{h k}-w\right\|_{B} \leq \widetilde{C}\left(h^{2}+k^{2}\right),
$$

from which it follows that $f_{h k}$ satisfies the compatibility condition (26).

Consider the problem

$$
\begin{aligned}
\delta z^{(i, j)} & =\sigma\left(x_{i}, y_{j}, z^{(i, j)}\right)+L_{1} u^{(i, j)}+L_{2} v^{(i, j)} \\
\delta_{2} u^{(i, j)} & =\sigma\left(x_{i}, y_{j}, z^{(i, j)}\right)+L_{1} u^{(i, j)}+L_{2} v^{(i, j)}, \\
\delta_{1} v^{(i, j)} & =\sigma\left(x_{i}, y_{j}, z^{(i, j)}\right)+L_{1} u^{(i, j)}+L_{2} v^{(i, j)},
\end{aligned}
$$

on $E_{h k}-\{(h, k)\}, E_{h k}-\{(0, k)\}, E_{h k}-\{(h, 0)\}$, respectively, and

$$
z^{(i, j)}=u^{(i, j)}=v^{(i, j)}=0, \quad \text { on } E_{h k}^{0} .
$$

We prove that the zero solution of this problem is stable.

Let $\left(\widetilde{z}_{h k}, \widetilde{u}_{h k}, \widetilde{v}_{h k}\right) \in \Xi_{+}$be a solution of the problem

$$
\begin{gathered}
\delta z^{(i, j)}=\sigma\left(x_{i}, y_{j}, z^{(i, j)}\right)+L_{1} u^{(i, j)}+L_{2} v^{(i, j)}+\alpha_{0}(h, k), \\
\delta_{2} u^{(i, j)}=\sigma\left(x_{i}, y_{j}, z^{(i, j)}\right)+L_{1} u^{(i, j)}+L_{2} v^{(i, j)}+\alpha_{1}(h, k), \\
\delta_{1} v^{(i, j)}=\sigma\left(x_{i}, y_{j}, z^{(i, j)}\right)+L_{1} u^{(i, j)}+L_{2} v^{(i, j)}+\alpha_{2}(h, k),
\end{gathered}
$$

on $E_{h k}-\{(h, k)\}, E_{h k}-\{(0, k)\}, E_{h k}-\{(h, 0)\}$, respectively, and

$$
z^{(i, j)}=\varepsilon_{0}(h, k), \quad u^{(i, j)}=\varepsilon_{1}(h, k), \quad v^{(i, j)}=\varepsilon_{2}(h, k), \quad \text { on } E_{h k}^{0},
$$

where $\alpha_{i}, \varepsilon_{i} \in \Theta, i=0,1,2$. Consider the Darboux problem

$$
\begin{aligned}
D_{x y} z(x, y)= & \sigma(x, y, z(x, y))+L_{1} D_{x} z(x, y)+L_{2} D_{y} z(x, y) \\
& +\alpha_{0}(h, k)+\alpha_{1}(h, k)+\alpha_{2}(h, k) \quad(x, y) \in \bar{E}, \\
z(x, 0)= & \varepsilon_{0}(h, k)+\varepsilon_{1}(h, k) x \quad \text { for } x \in[0, a], \\
z(0, y)= & \varepsilon_{0}(h, k)+\varepsilon_{2}(h, k) y \quad \text { for } y \in[0, b] .
\end{aligned}
$$


There is $\varrho>0$ such that if $h+k<\varrho$ then there exists a solution $z_{h k}: \bar{E} \rightarrow \mathbb{R}$ of this problem and

$$
\begin{aligned}
\lim _{(h, k) \rightarrow(0,0)} z_{h k}(x, y) & =\lim _{(h, k) \rightarrow(0,0)} D_{x} z_{h k}(x, y) \\
& =\lim _{(h, k) \rightarrow(0,0)} D_{y} z_{h k}(x, y)=0,
\end{aligned}
$$

uniformly on $\bar{E}$. It is obvious that the following functions are nondecreasing: $z_{h k}$ in $(x, y), D_{x} z_{h k}$ in $y$ and $D_{y} z_{h k}$ in $x$. The relations

$$
\begin{aligned}
D_{x} z_{h k}(x, y)= & \varepsilon_{1}(h, k) \\
& +\int_{0}^{y} e^{L_{1}(y-s)} \sigma(x, s, z(x, s)) d s+L_{2} \int_{0}^{y} e^{L_{1}(y-s)} D_{y} z_{h k}(x, s) d s, \\
D_{y} z_{h k}(x, y)= & \varepsilon_{2}(h, k) \\
& +\int_{0}^{x} e^{L_{2}(x-t)} \sigma(t, y, z(t, y)) d t+L_{1} \int_{0}^{x} e^{L_{2}(x-t)} D_{x} z_{h k}(t, y) d t
\end{aligned}
$$

yield that $D_{x} z_{h k}$ is nondecreasing in $x$ and $D_{y} z_{h k}$ in $y$. Thus for $h+k<\varrho$ we have

$$
\begin{gathered}
z_{h k}^{(i, j)}=\int_{0}^{x_{i}} \int_{0}^{y_{j}}\left[\sigma\left(t, s, z_{h k}(t, s)\right)+\begin{array}{r}
L_{1} D_{x} z_{h k}(t, s)+L_{2} D_{y} z_{h k}(t, s) \\
\left.+\alpha_{0}(h, k)+\alpha_{1}(h, k)+\alpha_{2}(h, k)\right] d t d s \\
+\varepsilon_{0}(h, k)+x_{i} \varepsilon_{1}(h, k)+y_{j} \varepsilon_{2}(h, k)
\end{array}\right. \\
\geq h k \sum_{\mu=0}^{i-1} \sum_{\nu=0}^{j-1}\left[\sigma\left(x_{\mu}, y_{\nu}, z_{h k}^{(\mu, \nu)}\right)+L_{1} D_{x} z_{h k}^{(\mu, \nu)}+L_{2} D_{y} z_{h k}^{(\mu, \nu)}\right. \\
\left.+\alpha_{0}(h, k)\right]+\varepsilon_{0}(h, k), \\
\left(D_{x} z_{h k}\right)^{(i, j)}=\int_{0}^{y_{j}}\left[\sigma\left(x_{i}, s, z_{h k}\left(x_{i}, s\right)\right)+L_{1} D_{x} z_{h k}\left(x_{i}, s\right)+L_{2} D_{y} z_{h k}\left(x_{i}, s\right)\right. \\
\left.+\alpha_{0}(h, k)+\alpha_{1}(h, k)+\alpha_{2}(h, k)\right] d s+\varepsilon_{1}(h, k) \\
+k \sum_{\nu=0}^{j-1}\left[\sigma\left(x_{i}, y_{\nu}, z_{h k}^{(i, \nu)}\right)+L_{1} D_{x} z_{h k}^{(i, \nu)}+L_{2} D_{y} z_{h k}^{(i, \nu)}\right. \\
\left(D_{y} z_{h k}\right)^{(i, j)}=\int_{0}^{x_{i}}\left[\sigma\left(t, y_{j}, z_{h k}\left(t, y_{j}\right)\right)+L_{1} D_{x} z_{h k}\left(t, y_{j}\right)+L_{2} D_{y} z_{h k}\left(t, y_{j}\right)\right. \\
\left.+\alpha_{0}(h, k)+\alpha_{1}(h, k)+\alpha_{2}(h, k)\right] d t+\varepsilon_{2}(h, k), \\
\geq h \sum_{\mu=0}^{i-1}\left[\sigma\left(x_{\mu}, y_{j}, z_{h k}^{(\mu, j)}\right)+L_{1} D_{x} z_{h k}^{(\mu, j)}+L_{2} D_{y} z_{h k}^{(\mu, j)}\right. \\
\left.+\alpha_{2}(h, k)\right]+\varepsilon_{2}(h, k),
\end{gathered}
$$


on $E_{h k}$. Since on the same set we have

$$
\begin{aligned}
& \widetilde{z}_{h k}^{(i, j)}=h k \sum_{\mu=0}^{i-1} \sum_{\nu=0}^{j-1}\left[\sigma\left(x_{\mu}, y_{\nu}, \widetilde{z}_{h k}^{(\mu, \nu)}\right)+L_{1} \widetilde{u}_{h k}^{(\mu, \nu)}+L_{2} \widetilde{v}_{h k}^{(\mu, \nu)}\right. \\
& \left.+\alpha_{0}(h, k)\right]+\varepsilon_{0}(h, k), \\
& \widetilde{u}_{h k}^{(i, j)}=k \sum_{\nu=0}^{j-1}\left[\sigma\left(x_{i}, y_{\nu}, \widetilde{z}_{h k}^{(i, \nu)}\right)+L_{1} \widetilde{u}_{h k}^{(i, \nu)}+L_{2} \widetilde{v}_{h k}^{(i, \nu)}+\alpha_{1}(h, k)\right]+\varepsilon_{1}(h, k), \\
& \widetilde{v}_{h k}^{(i, j)}=h \sum_{\mu=0}^{i-1}\left[\sigma\left(x_{\mu}, y_{j}, \widetilde{z}_{h k}^{(\mu, j)}\right)+L_{1} \widetilde{u}_{h k}^{(\mu, j)}+L_{2} \widetilde{v}_{h k}^{(\mu, j)}+\alpha_{2}(h, k)\right]+\varepsilon_{2}(h, k),
\end{aligned}
$$

it follows from Lemma 3 that $\widetilde{z}_{h k}^{(i, j)} \leq z_{h k}^{(i, j)}, \widetilde{u}_{h k}^{(i, j)} \leq\left(D_{x} z_{h k}\right)^{(i, j)}, \widetilde{v}_{h k}^{(i, j)} \leq$ $\left(D_{y} z_{h k}\right)^{(i, j)}$. Thus stability of $(31),(32)$ follows from (33).

4. Convergence of implicit difference methods. Let $K=1$ in the definition of $B_{h k}, B_{h k}^{1}, B_{h k}^{2}$. The operator $T_{h k}$ that we have used in the previous section may be defined by the same formula (29) on the sets $\left[-a_{0}, h\right] \times\left[-b_{0}, k\right],\left[-a_{0}, h\right] \times\left[-b_{0}, 0\right],\left[-a_{0}, 0\right] \times\left[-b_{0}, k\right]$. Using formula $(29)$ on these three domains respectively we define three new operators by

$$
\begin{array}{llrl}
S_{h k} w(t, s) & =T_{h k} w(t+h / 2, s+k / 2), & & (t, s) \in B, \\
\bar{S}_{h k} w(t, s) & =T_{h k} w(t+h / 2, s), & & (t, s) \in B, \\
\overline{\bar{S}}_{h k} w(t, s) & =T_{h k} w(t, s+k / 2), & & (t, s) \in B .
\end{array}
$$

Consider the difference problem

$$
\begin{aligned}
\delta z^{(i, j)} & =f\left(x_{i+1 / 2}, y_{j+1 / 2}, S_{h k} z_{[i, j]}, \bar{S}_{h k} u_{[i, j]}, \overline{\bar{S}}_{h k} v_{[i, j]}\right), \\
\delta_{2} u^{(i, j)} & =f\left(x_{i+1 / 2}, y_{i+1 / 2}, S_{h k} z_{[i, j]}, \bar{S}_{h k} u_{[i, j]}, \overline{\bar{S}}_{h k} v_{[i, j]}\right), \\
\delta_{1} v^{(i, j)} & =f\left(x_{i+1 / 2}, y_{j+1 / 2}, S_{h k} z_{[i, j]}, \bar{S}_{h k} u_{[i, j]}, \overline{\bar{S}}_{h k} v_{[i, j]}\right),
\end{aligned}
$$

on $E_{h k}-\{(h, k)\}, E_{h k}^{1}-\{(0, k)\}, E_{h k}^{2}-\{(h, 0)\}$, respectively, and

$$
z=\phi_{h k}, \quad u=\alpha_{h k}, \quad v=\beta_{h k}, \quad \text { on } E_{h k}^{0} .
$$

Assumption $\mathrm{H}_{4}$. Suppose that $f: \bar{E} \times C(B, \mathbb{R})^{3} \rightarrow \mathbb{R}$ is continuous and there are $L, L_{1}, L_{2} \in \mathbb{R}_{+}$such that

$$
\begin{aligned}
& \left|f\left(x, y, w, w_{1}, w_{2}\right)-f\left(x, y, \bar{w}, \bar{w}_{1}, \bar{w}_{2}\right)\right| \\
& \quad \leq L\|w-\bar{w}\|_{B}+L_{1}\left\|w_{1}-\bar{w}_{1}\right\|_{B}+L_{2}\left\|w_{2}-\bar{w}_{2}\right\|_{B} \quad \text { on } \bar{E} \times C(B, \mathbb{R})^{3} .
\end{aligned}
$$

TheOREM 6. Suppose that Assumption $\mathrm{H}_{4}$ is satisfied and

(i) $w: E^{0} \cup \bar{E} \rightarrow \mathbb{R}$ is a solution of problem (1),(2), and $w$ is of class $C^{4}$ on $\bar{E}$; 
(ii) $L a b+L_{1} b+L_{2} a<1$, and there are $\varepsilon_{i} \in \Theta, i=0,1,2$, such that inequalities (25) hold true.

Then there exists exactly one solution $\left(z_{h k}, u_{h k}, v_{h k}\right) \in \Xi$ of problem (34), (35) and there are $C, C_{1}, C_{2} \in \mathbb{R}_{+}$such that

$$
\begin{aligned}
\left|z_{h k}^{(i, j)}-w^{(i, j)}\right| & \leq x_{i} y_{j} \Upsilon(h, k)^{(i, j)}+x_{i} y_{j} C\left(h^{2}+k^{2}\right)+3 \varepsilon_{0}(h, k), \\
\left|u_{h k}^{(i, j)}-\left(D_{x} w\right)^{(i, j)}\right| & \leq y_{j} \Upsilon(h, k)^{(i+1, j)}+y_{j} C_{1}(h+k)+\varepsilon_{1}(h, k), \\
\left|v_{h k}^{(i, j)}-\left(D_{y} w\right)^{(i, j)}\right| & \leq x_{i} \Upsilon(h, k)^{(i, j+1)}+x_{i} C_{2}(h+k)+\varepsilon_{2}(h, k),
\end{aligned}
$$

on $E_{h k}, E_{h k}^{1}, E_{h k}^{2}$, respectively, where

$$
\begin{aligned}
\Upsilon(h, k)^{(i, j)}= & \frac{1}{1-L x_{i} y_{j}-L_{1} y_{j}-L_{2} x_{i}}\left[3 L \varepsilon_{0}(h, k)+L_{1} \varepsilon_{1}(h, k)\right. \\
& +L_{2} \varepsilon_{2}(h, k)+L x_{i} y_{j} C\left(h^{2}+k^{2}\right) \\
& \left.+L_{1} y_{j} C_{1}(h+k)+L_{2} x_{i} C_{2}(h+k)\right] .
\end{aligned}
$$

Pro of. Define

$$
f_{h k}\left(x_{i}, y_{j}, w, w_{1}, w_{2}\right)=f\left(x_{i+1 / 2}, y_{j+1 / 2}, S_{h k} w, \bar{S}_{h k} w_{1}, \overline{\bar{S}}_{h k} w_{2}\right) .
$$

Then

(36)

$$
\begin{aligned}
& \left|f_{h k}\left(x_{i}, y_{j}, w, w_{1}, w_{2}\right)-f_{h k}\left(x_{i}, y_{j}, \bar{w}, \bar{w}_{1}, \bar{w}_{2}\right)\right| \\
\leq & L|| S_{h k} w-S_{h k} \bar{w}\left\|_{B}+L_{1}\right\| \bar{S}_{h k} w_{1}-\bar{S}_{h k} \bar{w}_{1}\left\|_{B}+L_{2}\right\| \overline{\bar{S}}_{h k} w_{2}-\overline{\bar{S}}_{h k} \bar{w}_{2} \|_{B} \\
= & L|w-\bar{w}|_{h k}+L_{1}\left|w_{1}-\bar{w}_{1}\right|_{h k}+L_{2}\left|w_{2}-\bar{w}_{2}\right|_{h k} .
\end{aligned}
$$

Let

$$
\sigma_{h k}\left(x, y, w, w_{1}, w_{2}\right)=L|w|_{h k}+L_{1}\left|\bar{w}_{1}\right|_{h k}+L_{2}\left|\bar{w}_{2}\right|_{h k}
$$

for $\left(x, y, w, w_{1}, w_{2}\right) \in \bar{E}_{h k} \times \mathcal{F}\left[B_{h k}, \mathbb{R}_{+}\right] \times \mathcal{F}\left[B_{h k}^{1}, \mathbb{R}_{+}\right] \times \mathcal{F}\left[B_{h k}^{2}, \mathbb{R}_{+}\right]$. Then problem $(15),(16)$ with the above defined $\sigma_{h k}$ is equivalent to

$$
\begin{array}{cl}
z^{(i, j)}=h k \sum_{\mu=0}^{i-1} \sum_{\nu=0}^{j-1}\left[L z^{(\mu+1, \nu+1)}+L_{1} u^{(\mu, \nu+1)}+L_{2} v^{(\mu+1, \nu)}\right] & \text { on } E_{h k} \\
u^{(i, j)}=k \sum_{\nu=0}^{j-1}\left[L z^{(i+1, \nu+1)}+L_{1} u^{(i, \nu+1)}+L_{2} v^{(i+1, \nu)}\right] & \text { on } E_{h k}^{1} \\
v^{(i, j)}=h \sum_{\mu=0}^{i-1}\left[L z^{(\mu+1, j+1)}+L_{1} u^{(\mu, j+1)}+L_{2} v^{(\mu+1, j)}\right] & \text { on } E_{h k}^{2}, \\
z^{(i, j)}=u^{(i, j)}=v^{(i, j)}=0 \quad \text { on } E_{h k}^{0} . &
\end{array}
$$


It follows from (ii) that problem (37), (38) satisfies conditions (iii) and (iv) of Assumption $\mathrm{H}_{1}$. The uniqueness of the trivial solution of this problem follows from Remark 3.

Let $\eta_{h k}, \xi_{h k}, \lambda_{h k}$ be defined by

$$
\begin{gathered}
\delta w_{h k}^{(i, j)}=f\left(x_{i+1 / 2}, y_{j+1 / 2}, S_{h k}\left(w_{h k}\right)_{[i, j]},\right. \\
\left.\bar{S}_{h k}\left(D_{x} w_{h k}\right)_{[i, j]}, \overline{\bar{S}}_{h k}\left(D_{y} w_{h k}\right)_{[i, j]}\right)+\eta_{h k}^{(i, j)}, \\
\delta_{2}\left(D_{x} w_{h k}\right)^{(i, j)}=f\left(x_{i+1 / 2}, y_{j+1 / 2}, S_{h k}\left(w_{h k}\right)_{[i, j]},\right. \\
\left.\bar{S}_{h k}\left(D_{x} w_{h k}\right)_{[i, j]}, \overline{\bar{S}}_{h k}\left(D_{y} w_{h k}\right)_{[i, j]}\right)+\xi_{h k}^{(i, j)}, \\
\delta_{1}\left(D_{y} w_{h k}\right)^{(i, j)}=f\left(x_{i+1 / 2}, y_{j+1 / 2}, S_{h k}\left(w_{h k}\right)_{[i, j]},\right. \\
\left.\bar{S}_{h k}\left(D_{x} w_{h k}\right)_{[i, j]}, \overline{\bar{S}}_{h k}\left(D_{y} w_{h k}\right)_{[i, j]}\right)+\lambda_{h k}^{(i, j)} .
\end{gathered}
$$

From (i) and (30) it follows that there are $C, C_{1}, C_{2} \in \mathbb{R}_{+}$such that

$$
\left|\eta_{h k}^{(i, j)}\right| \leq C\left(h^{2}+k^{2}\right), \quad\left|\xi_{h k}^{(i, j)}\right| \leq C_{1}(h+k), \quad\left|\lambda_{h k}^{(i, j)}\right| \leq C_{2}(h+k) .
$$

Let

$$
\begin{gathered}
\widetilde{z}_{h k}^{(i, j)}=\max _{\substack{-M_{0} \leq \mu \leq i \\
-N_{0} \leq \nu \leq j}}\left|z_{h k}^{(\mu, \nu)}-w_{h k}^{(\mu, \nu)}\right|, \\
\widetilde{u}_{h k}^{(i, j)}=\max _{\substack{-M_{0} \leq \mu \leq i \\
-N_{0} \leq \nu \leq j}}\left|u_{h k}^{(\mu, \nu)}-D_{x} w_{h k}^{(\mu, \nu)}\right|, \quad \widetilde{v}_{h k}^{(i, j)}=\max _{\substack{-M_{0} \leq \mu \leq i \\
-N_{0} \leq \nu \leq j}}\left|v_{h k}^{(\mu, \nu)}-D_{y} w_{h k}^{(\mu, \nu)}\right| .
\end{gathered}
$$

Since these functions are nondecreasing we have by (36) the inequalities

$$
\begin{aligned}
\widetilde{z}_{h k}^{(i, j)} \leq h k \sum_{\mu=0}^{i-1} \sum_{\nu=0}^{j-1}\left[L \widetilde{z}_{h k}^{(\mu+1, \nu+1)}+L_{1} \widetilde{u}_{h k}^{(\mu, \nu+1)}+L_{2} \widetilde{v}_{h k}^{(\mu+1, \nu)}\right. & \left.+C\left(h^{2}+k^{2}\right)\right]+3 \varepsilon_{0}(h, k), \\
\widetilde{u}_{h k}^{(i, j)} \leq k \sum_{\nu=0}^{j-1}\left[L \widetilde{z}_{h k}^{(i+1, \nu+1)}+L_{1} \widetilde{u}_{h k}^{(i, \nu+1)}+\right. & L_{2} \widetilde{v}_{h k}^{(i+1, \nu)} \\
& \left.+C_{1}(h+k)\right]+\varepsilon_{1}(h, k), \\
\widetilde{v}_{h k}^{(i, j)} \leq h \sum_{\mu=0}^{i-1}\left[L \widetilde{z}_{h k}^{(\mu+1, j+1)}+L_{1} \widetilde{u}_{h k}^{(\mu, j+1)}+\right. & L_{2} \widetilde{v}_{h k}^{(\mu+1, j)} \\
& \left.+C_{2}(h+k)\right]+\varepsilon_{2}(h, k),
\end{aligned}
$$

on $E_{h k}, E_{h k}^{1}, E_{h k}^{2}$, respectively, and

$$
\widetilde{z}_{h k}^{(i, j)} \leq \varepsilon_{0}(h, k), \quad \widetilde{u}_{h k}^{(i, j)} \leq \varepsilon_{1}(h, k), \quad \widetilde{v}_{h k}^{(i, j)} \leq \varepsilon_{2}(h, k), \quad \text { on } E_{h k}^{0} .
$$


Let the functions $\overline{\bar{z}}_{h k}^{(i, j)}, \overline{\bar{u}}_{h k}^{(i, j)}, \overline{\bar{v}}_{h k}^{(i, j)}$, be defined by

(40) $\begin{aligned} \overline{\bar{u}}_{h k}^{(i, j)} & = \begin{cases}y_{j} \Upsilon(h, k)^{(i+1, j)}+y_{j} C_{1}(h+k)+\varepsilon_{1}(h, k) & \text { on } E_{h k}^{1}, \\ \varepsilon_{1}(h, k) & \text { on } E_{h k}^{0},\end{cases} \\ \overline{\bar{v}}_{h k}^{(i, j)} & = \begin{cases}x_{i} \Upsilon(h, k)^{(i, j+1)}+x_{i} C_{2}(h+k)+\varepsilon_{2}(h, k) & \text { on } E_{h k}^{1}, \\ \varepsilon_{2}(h, k) & \text { on } E_{h k}^{0}\end{cases} \end{aligned}$

We will prove by induction that

$$
\widetilde{z}_{h k}^{(i, j)} \leq \overline{\bar{z}}_{h k}^{(i, j)}, \quad \widetilde{u}_{h k}^{(i, j)} \leq \overline{\bar{u}}_{h k}^{(i, j)}, \quad \widetilde{v}_{h k}^{(i, j)} \leq \overline{\bar{v}}_{h k}^{(i, j)},
$$

on $E_{h k}, E_{h k}^{1}, E_{h k}^{2}$, respectively. The relations (41) obviously hold on $E_{h k}^{0}$. Suppose that for all $\mu, \nu$ such that $\mu<i, \nu<j$, we have

$$
\widetilde{z}_{h k}^{(\mu, \nu)} \leq \overline{\bar{z}}_{h k}^{(\mu, \nu)}, \quad \widetilde{u}_{h k}^{(\mu-1, \nu)} \leq \overline{\bar{u}}_{h k}^{(\mu-1, \nu)}, \quad \widetilde{v}_{h k}^{(\mu, \nu-1)} \leq \overline{\bar{v}}_{h k}^{(\mu, \nu-1)} .
$$

By (39) we see that $\widetilde{z}_{h k}, \widetilde{u}_{h k}, \widetilde{v}_{h k}$ satisfy the inequalities

$$
\begin{aligned}
z^{(i, j)}- & h k\left[L z^{(i, j)}+L_{1} u^{(i-1, j)}+L_{2} v^{(i, j-1)}\right] \\
\leq & h k \sum_{\substack{\mu=0 \\
(\mu, \nu) \neq(i-1, j-1)}}^{j-1}\left[L z^{(\mu+1, \nu+1)}+L_{1} u^{(\mu, \nu+1)}+L_{2} v^{(\mu+1, \nu)}+C\left(h^{2}+k^{2}\right)\right]+3 \varepsilon_{0}(h, k), \\
u^{(i-1, j)}- & k\left[L z^{(i, j)}+L_{1} u^{(i-1, j)}+L_{2} v^{(i, j-1)}\right] \quad \\
\leq & k \sum_{\nu=0}^{j-2}\left[L z^{(i, \nu+1)}+L_{1} u^{(i-1, \nu+1)}+L_{2} v^{(i, \nu)}+C_{1}(h+k)\right]+\varepsilon_{1}(h, k), \\
v^{(i, j-1)} & -h\left[L z^{(i, j)}+L_{1} u^{(i-1, j)}+L_{2} v^{(i, j-1)}\right] \\
\leq & h \sum_{\mu=0}^{i-2}\left[L z^{(\mu+1, j)}+L_{1} u^{(\mu, j)}+L_{2} v^{(\mu+1, j-1)}+C_{2}(h+k)\right]+\varepsilon_{2}(h, k),
\end{aligned}
$$

while the functions $\overline{\bar{z}}_{h k}, \overline{\bar{u}}_{h k}, \overline{\bar{v}}_{h k}$ defined by (40) satisfy the reverse inequalities. Thus by the inductive assumption we get

$$
\begin{aligned}
\widetilde{z}_{h k}^{(i, j)}-h k\left[L \widetilde{z}_{h k}^{(i, j)}+\right. & \left.L_{1} \widetilde{u}_{h k}^{(i-1, j)}+L_{2} \widetilde{v}_{h k}^{(i, j-1)}\right] \\
& \leq \overline{\bar{z}}_{h k}^{(i, j)}-h k\left[L \overline{\bar{z}}_{h k}^{(i, j)}+L_{1} \overline{\bar{u}}_{h k}^{(i-1, j)}+L_{2} \overline{\bar{v}}_{h k}^{(i, j-1)}\right], \\
\widetilde{u}_{h k}^{(i-1, j)}-k\left[L \widetilde{z}_{h k}^{(i, j)}+\right. & \left.L_{1} \widetilde{u}_{h k}^{(i-1, j)}+L_{2} \widetilde{v}_{h k}^{(i, j-1)}\right] \\
& \leq \overline{\bar{u}}_{h k}^{(i-1, j)}-k\left[L \overline{\bar{z}}_{h k}^{(i, j)}+L_{1} \overline{\bar{u}}_{h k}^{(i-1, j)}+L_{2} \overline{\bar{v}}_{h k}^{(i, j-1)}\right],
\end{aligned}
$$




$$
\begin{aligned}
\widetilde{v}_{h k}^{(i, j-1)}-h\left[L \widetilde{z}_{h k}^{(i, j)}+\right. & \left.L_{1} \widetilde{u}_{h k}^{(i-1, j)}+L_{2} \widetilde{v}_{h k}^{(i, j-1)}\right] \\
& \leq \overline{\bar{v}}_{h k}^{(i, j-1)}-h\left[L \overline{\bar{z}}_{h k}^{(i, j)}+L_{1} \overline{\bar{u}}_{h k}^{(i-1, j)}+L_{2} \overline{\bar{v}}_{h k}^{(i, j-1)}\right],
\end{aligned}
$$

from which we obtain

$$
\widetilde{z}_{h k}^{(i, j)} \leq \overline{\bar{z}}_{h k}^{(i, j)}, \quad \widetilde{u}_{h k}^{(i-1, j)} \leq \overline{\bar{u}}_{h k}^{(i-1, j)}, \quad \widetilde{v}_{h k}^{(i, j-1)} \leq \overline{\bar{v}}_{h k}^{(i, j-1)} .
$$

Thus (41) follows by induction, which completes the proof of Theorem 6 .

5. Numerical examples. Equation (3) with deviated argument is an example of a functional equation (1). As a special case of (3) consider the equation

$$
D_{x y} z(x, y)=z(x, y)-x D_{x} z(x / 2, y)-y D_{y} z(x, y / 2)+g_{1}(x, y)
$$

on $E=[0,1 / 2] \times[0,1 / 2]$, where

$$
g_{1}(x, y)=1+4 x y+3 y^{2}+x^{2} y^{2}+\frac{3}{4} x y^{3} .
$$

Since the delays $x / 2$ and $y / 2$ are nonnegative for $(x, y) \in E$, for the initial set $E^{0}$ we may take $[0,1 / 2] \times\{0\} \cup\{0\} \times[0,1 / 2]$. For the Darboux condition for (42) we take

(43) $z(x, 0)=-1+x \quad$ for $x \in[0,1 / 2], \quad z(0, y)=-1 \quad$ for $y \in[0,1 / 2]$.

The explicit difference scheme (27), (28) for (42), (43) takes the form

$$
\begin{gathered}
\delta z^{(i, j)}=z^{(i, j)}-x_{i} T_{h k} u\left(x_{i} / 2, y_{j}\right)-y_{j} T_{h k} v\left(x_{i}, y_{j} / 2\right)+g_{1}\left(x_{i}, y_{j}\right), \\
\delta_{2} u^{(i, j)}=z^{(i, j)}-x_{i} T_{h k} u\left(x_{i} / 2, y_{j}\right)-y_{j} T_{h k} v\left(x_{i}, y_{j} / 2\right)+g_{1}\left(x_{i}, y_{j}\right), \\
\delta_{1} v^{(i, j)}=z^{(i, j)}-x_{i} T_{h k} u\left(x_{i} / 2, y_{j}\right)-y_{j} T_{h k} v\left(x_{i}, y_{j} / 2\right)+g_{1}\left(x_{i}, y_{j}\right), \\
z^{(i, j)}=-1+x_{i}, \quad u^{(i, j)}=1, \quad v^{(i, j)}=0 \quad \text { if } j=0, \\
z^{(i, j)}=-1, \quad u^{(i, j)}=1+y_{j}^{3}, \quad v^{(i, j)}=0 \quad \text { if } i=0 .
\end{gathered}
$$

Note that

$$
\begin{aligned}
& T_{h k} u\left(x_{i} / 2, y_{j}\right)= \begin{cases}u^{(i / 2, j)} & \text { if } i \text { is even, } \\
\frac{1}{2}\left(u^{((i-1) / 2, j)}+u^{((i+1) / 2, j)}\right) & \text { if } i \text { is odd, }\end{cases} \\
& T_{h k} v\left(x_{i}, y_{j} / 2\right)= \begin{cases}v^{(i, j / 2)} & \text { if } j \text { is even, } \\
\frac{1}{2}\left(v^{(i,(j-1) / 2)}+v^{(i,(j+1) / 2)}\right) & \text { if } j \text { is odd. }\end{cases}
\end{aligned}
$$

The exact solution to $(42),(43)$ is $w(x, y)=-1+x+x^{2} y^{2}+x y^{3}$. If $h=k=$ 0.005 then the diagonal values of the errors at every tenth diagonal knot of our mesh are given in Table 1. 
Table 1

\begin{tabular}{cccc}
\hline$x_{i}=y_{i}$ & $z^{(i, i)}-w\left(x_{i}, x_{i}\right)$ & $u^{(i, i)}-D_{x} w\left(x_{i}, x_{i}\right)$ & $v^{(i, i)}-D_{y} w\left(x_{i}, x_{i}\right)$ \\
\hline 0.05 & -0.00000209 & -0.00004312 & -0.00002500 \\
0.10 & -0.00001712 & -0.00017374 & -0.00009999 \\
0.15 & -0.00005822 & -0.00039185 & -0.00022496 \\
0.20 & -0.00013849 & -0.00069744 & -0.00039990 \\
0.25 & -0.00027108 & -0.00109051 & -0.00062481 \\
0.30 & -0.00046910 & -0.00157105 & -0.00089967 \\
0.35 & -0.00074566 & -0.00213905 & -0.00122447 \\
0.40 & -0.00111390 & -0.00279452 & -0.00159923 \\
0.45 & -0.00158694 & -0.00353745 & -0.00202388 \\
0.50 & -0.00217789 & -0.00436783 & -0.00249852 \\
\hline
\end{tabular}

If for comparison we consider the implicit difference scheme (34), (35) for problem (42), (43) then we get

$$
\begin{aligned}
& \delta z^{(i, j)}= S_{h k} z\left(x_{i}, y_{j}\right)-x_{i+1 / 2} \bar{S}_{h k} u\left(x_{i} / 2, y_{j}\right) \\
&-y_{j+1 / 2} \overline{\bar{S}}_{h k} v\left(x_{i}, y_{j} / 2\right)+g_{1}\left(x_{i+1 / 2}, y_{j+1 / 2}\right), \\
& \delta_{2} u^{(i, j)}= S_{h k} z\left(x_{i}, y_{j}\right)-x_{i+1 / 2} \bar{S}_{h k} u\left(x_{i} / 2, y_{j}\right) \\
& \quad-y_{j+1 / 2} \overline{\bar{S}}_{h k} v\left(x_{i}, y_{j} / 2\right)+g_{1}\left(x_{i+1 / 2}, y_{j+1 / 2}\right), \\
& \delta_{1} v^{(i, j)}= S_{h k} z\left(x_{i}, y_{j}\right)-x_{i+1 / 2} \bar{S}_{h k} u\left(x_{i} / 2, y_{j}\right) \\
&-y_{j+1 / 2} \overline{\bar{S}}_{h k} v\left(x_{i}, y_{j} / 2\right)+g_{1}\left(x_{i+1 / 2}, y_{j+1 / 2}\right), \\
& z^{(i, j)}=-1+ x_{i}, \quad u^{(i, j)}=1, \quad v^{(i, j)}=0, \quad \text { if } j=0, \\
& z^{(i, j)}=-1, \quad u^{(i, j)}=1+y_{j}^{3}, \quad v^{(i, j)}=0, \quad \text { if } i=0 .
\end{aligned}
$$

In this case we have

$$
\begin{aligned}
S_{h k} z\left(x_{i}, y_{j}\right) & =\frac{1}{4}\left(z^{(i, j)}+z^{(i+1, j)}+z^{(i, j+1)}+z^{(i+1, j+1)}\right), \\
\bar{S}_{h k} u\left(x_{i} / 2, y_{j}\right) & = \begin{cases}\frac{1}{2}\left(u^{(i / 2, j)}+u^{(i / 2+1, j)}\right) & \text { if } i \text { is even, } \\
u^{((i+1) / 2, j)} & \text { if } i \text { is odd, }\end{cases} \\
\overline{\bar{S}}_{h k} v\left(x_{i}, y_{j} / 2\right) & = \begin{cases}\frac{1}{2}\left(v^{(i, j / 2)}+v^{(i, j / 2+1)}\right) & \text { if } j \text { is even, } \\
v^{(i,(j+1) / 2)} & \text { if } j \text { is odd. }\end{cases}
\end{aligned}
$$

The errors for $h=k=0.005$ are given in Table 2 .

As an example of a differential-integral equation (4) consider the equation

$$
\begin{aligned}
& D_{x y} z(x, y)=D_{x} z(x, y)+2 D_{y} z(x, y) \\
& +16 \int_{x-1 / 2}^{x} \int_{y-1 / 2}^{y} z(t, s) d t d s+g_{2}(x, y) \quad \text { on } E=[0,1 / 2] \times[0,1 / 2]
\end{aligned}
$$


Table 2

\begin{tabular}{cccc}
\hline$x_{i}=y_{i}$ & $z^{(i, i)}-w\left(x_{i}, x_{i}\right)$ & $u^{(i, i)}-D_{x} w\left(x_{i}, x_{i}\right)$ & $v^{(i, i)}-D_{y} w\left(x_{i}, x_{i}\right)$ \\
\hline 0.05 & -0.00000002 & 0.00001217 & 0.00005093 \\
0.10 & -0.00000007 & 0.00004910 & 0.00020171 \\
0.15 & -0.00000021 & 0.00011015 & 0.00045192 \\
0.20 & -0.00000054 & 0.00019426 & 0.00080080 \\
0.25 & -0.00000128 & 0.00029996 & 0.00124732 \\
0.30 & -0.00000280 & 0.00042537 & 0.00179014 \\
0.35 & -0.00000564 & 0.00056822 & 0.00242765 \\
0.40 & -0.00001055 & 0.00072584 & 0.00315796 \\
0.45 & -0.00001852 & 0.00089523 & 0.00397896 \\
0.50 & -0.00003081 & 0.00107304 & 0.00488826 \\
\hline
\end{tabular}

where $g_{2}(x, y)=5-9 y+4 x y+24 x y^{2}$, together with the Darboux condition

$$
\begin{aligned}
z(x, y)= & -1+2 x y-6 x y^{2} \\
& \text { on } E^{0}=[-1 / 2,1 / 2] \times[-1 / 2,1 / 2] \backslash(0,1 / 2] \times(0,1 / 2] .
\end{aligned}
$$

The explicit difference scheme (27), (28) for (44), (45) takes the form

$$
\begin{aligned}
& \delta z^{(i, j)}=u^{(i, j)}+2 v^{(i, j)}+16 \int_{x-1 / 2}^{x} \int_{y-1 / 2}^{y} T_{h k} z(t, s) d t d s+g_{2}\left(x_{i}, y_{j}\right), \\
& \delta_{2} u^{(i, j)}=u^{(i, j)}+2 v^{(i, j)}+16 \int_{x-1 / 2}^{x} \int_{y-1 / 2}^{y} T_{h k} z(t, s) d t d s+g_{2}\left(x_{i}, y_{j}\right), \\
& \delta_{1} v^{(i, j)}=u^{(i, j)}+2 v^{(i, j)}+16 \int_{x-1 / 2}^{x} \int_{y-1 / 2}^{y} T_{h k} z(t, s) d t d s+g_{2}\left(x_{i}, y_{j}\right),
\end{aligned}
$$

and

$$
z^{(i, j)}=-1+2 x_{i} y_{j}-6 x_{i} y_{j}^{2}, \quad u^{(i, j)}=2 y_{j}-6 y_{j}^{2}, \quad v^{(i, j)}=2 x_{i}-12 x_{i} y_{j},
$$

on $E_{h k}^{0}$. Note that the integrals in the above scheme may be computed easily if we use the relation

$$
\int_{x_{i}}^{x_{i+1}} \int_{y_{j}}^{y_{j+1}} T_{h k} z(t, s) d t d s=\frac{h k}{2}\left(z^{(i, j)}+z^{(i+1, j)}+z^{(i, j+1)}+z^{(i+1, j+1)}\right) .
$$

The exact solution to $(42),(43)$ is $w(x, y)=-1+2 x y-6 x y^{2}$. The errors for $h=k=0.005$ are given in Table 3 . 
Table 3

\begin{tabular}{cccc}
\hline$x_{i}=y_{i}$ & $z^{(i, i)}-w\left(x_{i}, x_{i}\right)$ & $u^{(i, i)}-D_{x} w\left(x_{i}, x_{i}\right)$ & $v^{(i, i)}-D_{y} w\left(x_{i}, x_{i}\right)$ \\
\hline 0.05 & 0.00007685 & 0.00153899 & 0.00008161 \\
0.10 & 0.00031647 & 0.00318277 & 0.00035100 \\
0.15 & 0.00073559 & 0.00497368 & 0.00085878 \\
0.20 & 0.00135639 & 0.00697171 & 0.00167348 \\
0.25 & 0.00220865 & 0.00926232 & 0.00288914 \\
0.30 & 0.00333269 & 0.01196712 & 0.00463545 \\
0.35 & 0.00478371 & 0.01525837 & 0.00709152 \\
0.40 & 0.00663757 & 0.01937877 & 0.01050441 \\
0.45 & 0.00899891 & 0.02466848 & 0.01521429 \\
0.50 & 0.01201233 & 0.03160205 & 0.02168859 \\
\hline
\end{tabular}

\section{References}

[1] P. Brandi, Z. Kamont and A. Salvadori, Approximate solutions of mixed problems for first order partial differential-functional equations, Atti Sem. Mat. Fis. Univ. Modena 39 (1991), 277-302.

[2] T. Człapiński, Existence of solutions of the Darboux problem for partial differential-functional equations with infinite delay in a Banach space, Comment. Math. 35 (1995), 111-122.

[3] Z. Denkowski and A. Pelczar, On the existence and uniqueness of solutions of some partial differential functional equations, Ann. Polon. Math. 35 (1978), 261304.

[4] Z. Kamont, Finite difference approximations for first-order partial differentialfunctional equations, Ukrain. Math. J. 46 (1994), 265-287.

[5] Z. Kamont and M. Kwapisz, On the Cauchy problem for differential-delay equations in a Banach space, Math. Nachr. 74 (1976), 173-190.

[6] _ - - On non-linear Volterra integral-functional equations in several variables, Ann. Polon. Math. 40 (1981), 1-29.

[7] Z. Kamont and H. Leszczyński, Stability of difference equations generated by parabolic differential-functional problems, Rend. Mat. 16 (1996), 265-287.

[8] - - - Numerical solutions to the Darboux problem with the functional dependence, Georgian Math. J. 5 (1998), 71-90.

[9] H. Leszczyński, Convergence of one-step difference methods for nonlinear parabolic differential-functional systems with initial-boundary conditions of the Dirichlet type, Comment. Math. 30 (1990), 357-375.

[10] - Convergence of difference analogues to the Darboux problem with functional dependence, Bull. Belgian Math. Soc. 5 (1998), 39-57.

[11] M. Malec, C. Mączka and W. Voigt, Weak difference-functional inequalities and their applications to the difference analogue of non-linear parabolic differentialfunctional equations, Beiträge Numer. Math. 11 (1983), 69-79.

[12] M. Malec and M. Rosati, Weak monotonicity for non linear systems of functionalfinite difference inequalities of parabolic type, Rend. Mat. 3 (1983), 157-170.

[13] M. Malec et A. Schiaffino, Méthode aux différence finies pour une équation nonlinéaire différentielle fonctionnelle du type parabolique avec une condition initiale de Cauchy, Boll. Un. Mat. Ital. 7B (1987), 99-109. 
[14] A. Pelczar, Some functional-differential equations, Dissertationes Math. 100 (1973).

[15] T. Ważewski, Sur une extension du procédé de I. Jungermann pour établir la convergence des approximations successives au cas des équations différentielles ordinaires, Bull. Acad. Polon. Sci. Sér. Sci. Math. Astronom. Phys. 8 (1960), 43-46.

[16] M. Zennaro, Delay differential equations: theory and numerics, in: Theory and Numerics of Ordinary and Partial Differential Equations, Clarendon Press, Oxford, 1995, 291-333.

Institute of Mathematics

University of Gdańsk

Wita Stwosza 57

80-952 Gdańsk, Poland

E-mail: czltsz@ksinet.univ.gda.pl

Reçu par la Rédaction le 11.5.1998 